\title{
Developing Liquid Protection Schemes for Fusion Energy Reactor First Walls
}

Principal Investigator:

Co-Principal Investigators:

Reporting Period:

DOE Contract Number:
Dr. Minami Yoda, Associate Professor

Woodruff School of Mechanical Engineering

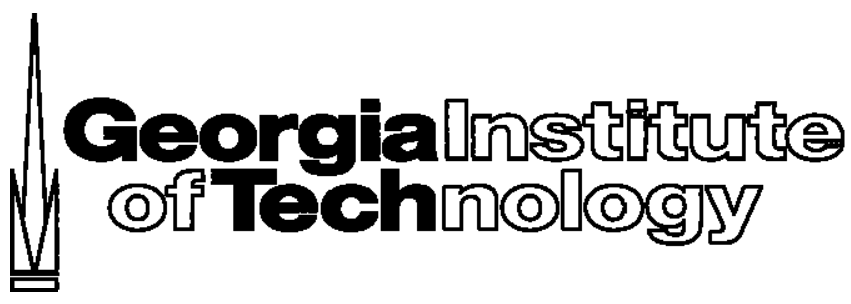

Atlanta, GA 30332-0405 USA

minami.yoda@me.gatech.edu

Dr. S. I. Abdel-Khalik, SNC Distinguished Prof. said.abdelkhalik@me.gatech.edu

January 1 - December 31, 2005

DE-FG02-98ER54499 


\section{Experimental Studies of Turbulent Liquid Sheets}

Over the last year, the Georgia Tech group has experimentally studied vertical turbulent sheets of water issuing downwards into atmospheric pressure air at Reynolds numbers $R e$ $=U_{\mathrm{o}} \delta / \nu=53,000$ and 120,000 and Weber numbers $W e=\rho U_{\mathrm{o}}{ }^{2} \delta / \sigma=2,900$ and 18,000, respectively. Here, $U_{0}$ is the average jet speed, $\delta$ is the jet thickness (short dimension) at the nozzle exit $(\delta=1 \mathrm{~cm})$, and $\nu, \rho$ and $\sigma$ are the kinematic viscosity and density of water and the surface tension at the air-water interface, respectively. These $R e$ and $W e$ values are about $50 \%$ and $20 \%$ of the prototypical values for HYLIFE-II, respectively. In this report, the flow coordinate system is defined so that the origin is at the center of the nozzle exit, with the $x$-axis along the flow direction, the $y$-axis along the long dimension of the nozzle, and the z-axis along the short dimension of the nozzle (cf. Fig. 1).

During the final year of this project, we have made three contributions in the area of thermal-hydraulics of thick liquid protection, namely:

1) Experimentally demonstrated that removing as little as $1 \%$ of the total mass flux using boundary-layer (BL) cutting can reduce the number density of the drops due to turbulent breakup of the liquid sheet below the maximum background density levels recommended for HYLIFE-II of $5 \times 10^{-19} \mathrm{~m}^{3} ;{ }^{1}$

2) Shown that a well-designed flow conditioning section upstream of the nozzle can greatly reduce surface ripple, and that boundary-layer cutting can be used in conjunction with well-designed flow conditioning to further reduce surface ripple below the $0.07 \delta$ beam-to-jet standoff proposed for HYLIFE-II; ${ }^{2}$

3) Quantified how different flow conditioner designs affect the rms fluctuations of the streamwise $(x)$ and transverse $(z)$ velocity components in the nozzle itself (i.e., upstream of the nozzle exit) and affect surface ripple in the near-field of the flow, or $x \leq 25 \delta$.

The rest of this section details these conclusions. In all cases, further details of this work can be found in the doctoral dissertation by Durbin. ${ }^{3}$

The flow conditioner (Fig. 1) in all cases consisted of a stainless steel perforated plate, PP (50\% open area ratio, $4.8 \mathrm{~mm}$ diameter staggered holes), followed by a $2.5 \mathrm{~cm}$ section of polycarbonate honeycomb, HC (circular cells of diameter $0.32 \mathrm{~cm}$ ), and a fine screen, FS $(30 \times 30$ mesh, $37.1 \%$ open area with $0.33 \mathrm{~mm}$ diameter wires spaced $0.51 \mathrm{~mm}$ apart). The edge-toedge $x$-distances between the PP and $\mathrm{HC}$ and the $\mathrm{HC}$ and FS were $3.9 \mathrm{~cm}$ and $0.5 \mathrm{~cm}$, respectively. The

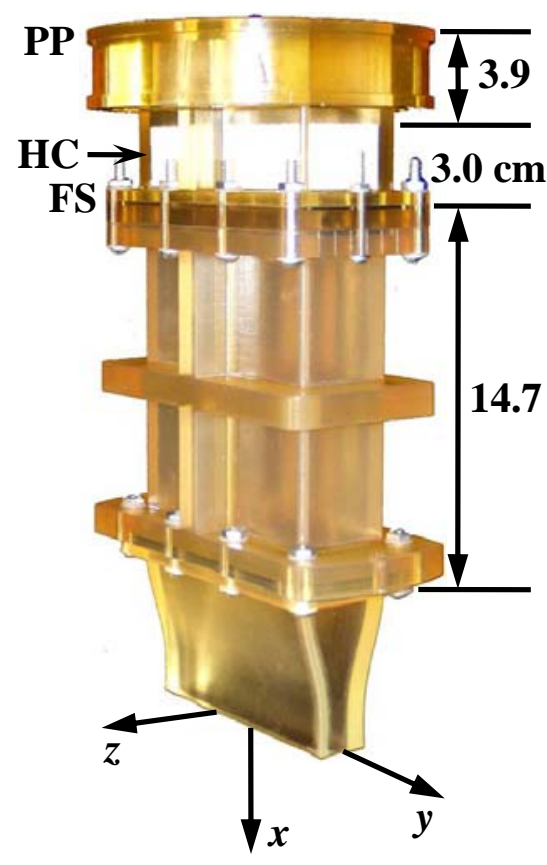

Figure 1 Photo of flow conditioner consisting of perforated plate (PP), honeycomb (HC) and fine screen (FS) with nozzle. 
results using this "baseline" flow conditioner were compared with those obtained using the same flow conditioner assembly without a FS.

The BL cutter used in this study removed fluid from only one face of the flow along the $y$-axis just downstream of the nozzle exit at $x=0.076 \mathrm{~cm}$. The cutter position could be adjusted along the $z$-direction to control the level of BL cutting so that the mass flow rate diverted by the cutter, $\dot{m}_{\text {cut }}$, varied from 0 to $1.9 \%$ of the total flow rate, or $\dot{m}_{\mathrm{fl}}=10.82$ $\mathrm{kg} / \mathrm{s}$, corresponding to $R e=120,000$. The aluminum cutter blade had a width (y-extent) of $12 \mathrm{~cm}$ that extended $1 \mathrm{~cm}$ beyond the jet on both sides.

Planar laser-induced fluorescence (PLIF) was used to obtain the free-surface geometry and calculate the average and standard deviation of the z-position of the free surface. The turbulent sheet of water dyed with $26 \mathrm{mg} / \mathrm{L}$ disodium fluorescein was illuminated at various downstream $(x)$ locations by a $\sim 2 \mathrm{~mm}$ thick laser light sheet, and the resultant interface between fluorescing water and non-fluorescing air (which corresponds to the free surface) was imaged obliquely from below. The z-position of the free surface, which was determined using a threshold-based edge detection scheme, was visualized using up to five overlapping segments that span one side of the entire $y$-extent of the flow.

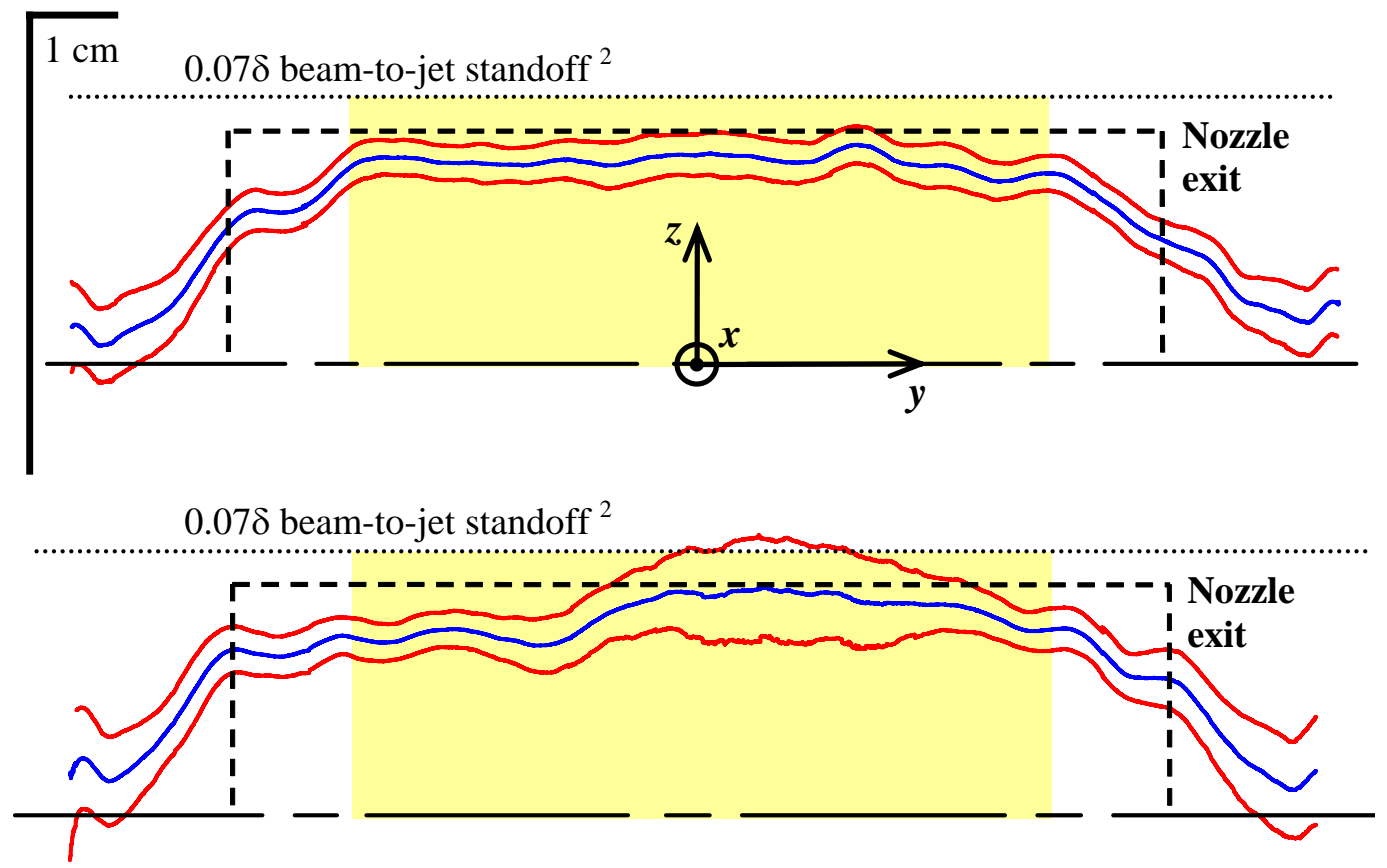

Figure 2 PLIF results showing the average $z$-position of the free surface in blue of the turbulent liquid sheet at $x / \delta=25$ for $R e=120,000$ for the baseline flow conditioner with [top] and without [bottom] the fine screen. The curves in red represent one standard deviation above and below this mean.

Figure 2 shows the average free-surface z-position (blue) and one standard deviation in zposition above and below this average (red) obtained using PLIF for the flow conditioner with [top] and without [bottom] the screen at $x / \delta=25$ for the turbulent liquid sheet at $R e$ $=120,000$. Only the upper half of the flow is shown, with the flow centerline at the bottom. The (half-)nozzle exit with a vertical extent of $0.5 \mathrm{~cm}$ (i.e., $0.5 \delta$ ) is indicated by 
the heavy dashed rectangle, while the beam-to-jet standoff of $0.07 \delta$ is indicated by the dashed line. Note that the vertical magnification is five times the horizontal magnification. The liquid sheet issuing from the flow conditioner without the screen has much greater surface ripple, even this far downstream from the nozzle exit, with a strong "bulge" near the center. Clearly, this flow configuration cannot satisfy the beam-to-jet standoff proposed for HYLIFE-II for even a modest limit of one standard deviation about the mean.

Given that the fluctuations in free-surface z-position are fairly consistent over the central portion of the flow, these values were spatially averaged over the central $75 \%$ of the $y$ extent of the nozzle (the shaded region in Fig. 2). Figure 3 shows this averaged standard deviation, $\sigma_{z}$, normalized by the nozzle thickness, $\delta$, as a function of normalized distance downstream of the nozzle $x / \delta$, for the flow conditioner with (black symbols) and without (gray symbols) a screen. Closed and open symbols denote no cutting and BL cutting at $\dot{m}_{\text {cut }} / \dot{m}_{\mathrm{fl}}=1.9 \%$, respectively. BL cutting clearly reduces surface ripple, but the addition of the fine screen reduces surface ripple much more than even this maximum level of BL cutting. BL cutting appears to also give a greater reduction in surface ripple for flow conditioner with (vs. without) the fine screen.

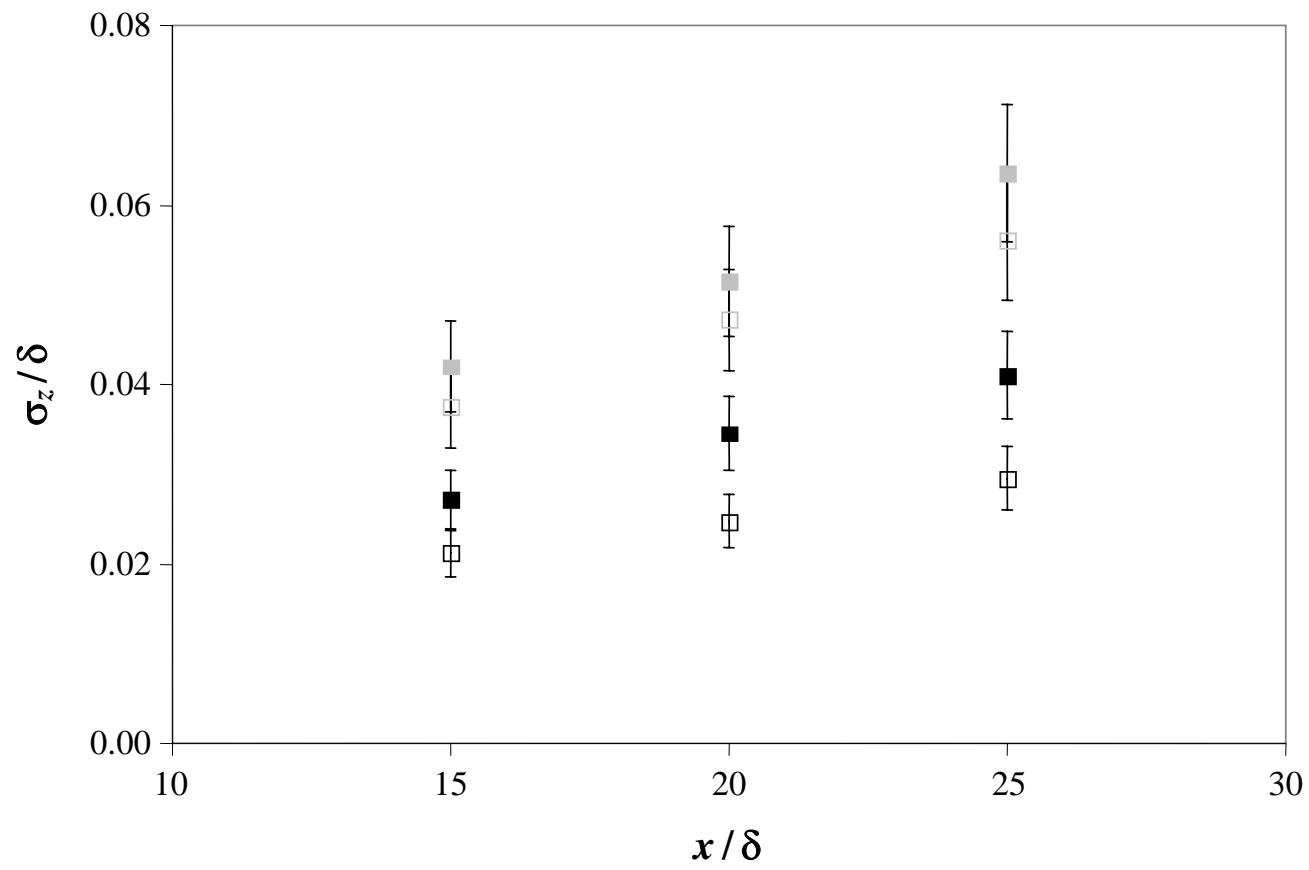

Figure 3 Plot of the spatially averaged standard deviation in the z-position of the free surface as a function of $x$-position at $R e=120,000$, both normalized by the nozzle thickness, for the flow conditioner with (black) and without (gray symbols) a screen. Closed and open symbols indicate no cutting and BL cutting at $1.9 \%$ of the total mass flow rate $\dot{m}_{\mathrm{fl}}$.

The number density of ejected drops due to turbulent breakup of the flow was estimated by mass collection. The ejected fluid was collected by a row of five square cuvettes tilted towards the free-surface at $6.5^{\circ}$ with respect to the vertical and centered with respect to the $y$-axis just beyond the free surface at the furthest downstream location of 
$x / \delta=25$. The row was positioned at various cuvette standoff distances $\Delta z_{\mathrm{s}}$ measured from the nearest inner wall of the nozzle, or the nominal jet free surface. The cuvettes were weighed on a digital scale before and after each 30-60 minute collection period; the resultant mass difference then gave an estimate of the mass of drops ejected from the free surface.

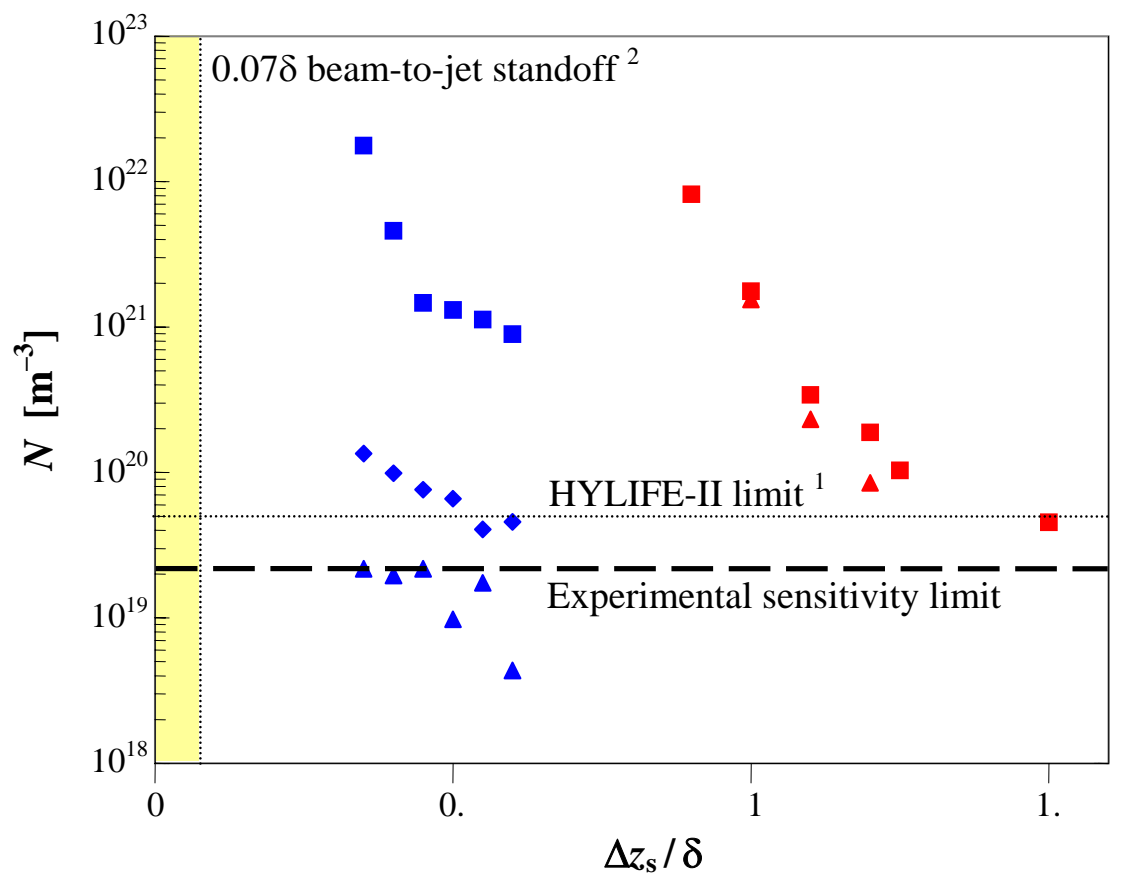

Figure 4 Equivalent number density $N$ of ejected drops as a function of normalized cuvette standoff distance $\Delta z_{\mathrm{s}} / \delta$ with BL cutting at $0 \%(\boldsymbol{\nabla}), 1 \%(\diamond)$ and $1.9 \%(\boldsymbol{\Delta})$ of the total mass flow rate $\dot{m}_{\mathrm{fl}}$. The blue and red symbols denote results for the flow conditioner with and without the screen, respectively.

Figure 4 shows a plot of the equivalent number density $N$ (log scale) for the drops ejected due to turbulent breakup of the liquid sheet at $R e=120,000$ calculated from the mass collection results as a function of the cuvette standoff distance normalized by the sheet thickness for the standard flow conditioner with (blue symbols) and without (red symbols) a fine screen. Squares, diamonds and triangles correspond to $\dot{m}_{\text {cut }} / \dot{m}_{\mathrm{fl}}=0$ (no cutting), $1 \%$ and $1.9 \%$, respectively. Note that all data at or below the indicated experimental sensitivity limit (derived from the sensitivity limits of the digital scale of 1 $\mathrm{mg} /$ cuvette, or $5 \mathrm{mg}$ total collected over 30 minutes) are considered to indicate a collected mass of zero within experimental error. The confidence intervals for these data are estimated to be about $40 \%$. In all cases, the ejected drop density increases as we approach the free surface. Boundary-layer cutting reduces $N$ and presumably turbulent breakup of the liquid sheet; breakup appears to be essentially suppressed (within experimental error) for the baseline flow conditioner at $\dot{m}_{\text {cut }} / \dot{m}_{\mathrm{fl}}=1.9 \%$. More drops are found farther from the free surface for the flow conditioner without the screen, in part because this configuration has greater surface ripple ( $c f$. Fig. 2). BL cutting also appears to have less impact upon the flow conditioner without fine screen, giving smaller reductions in $N$ compared with the corresponding cases for the baseline flow conditioner. 
Overall, the (presence or absence of the) fine screen has a much greater impact on turbulent breakup than BL cutting, and BL cutting appears to only be successful at suppressing this breakup for a "well-conditioned" flow. These results, in conjunction with those for surface ripple, clearly demonstrate that both well-designed flow conditioning and BL cutting will be required to create thick liquid protection schemes that will meet current design specifications.

Finally, laser-Doppler velocimetry (LDV) was used to quantify the streamwise $(x)$ and transverse (z) velocity components. Two of the liquid sheet nozzles fabricated using stereolithography rapid prototyping were modified to include Lexan (polycarbonate) and glass windows for optical access through the side (short dimension) of the nozzle. Profiles of mean velocity and rms velocity fluctuations for the components along $x$ (streamwise) and $z$ (cross-stream), or $\left(u, u^{\prime}\right)$ and $\left(w, w^{\prime}\right)$, respectively, were acquired using a single-component LDV system (VioSense MiniLDV-80) in backscatter mode with a working distance of $80 \mathrm{~mm}$ and a probe volume with FWHM dimensions of $100 \times$ $1200 \times 40 \mu \mathrm{m}(x \times y \times z)$ (Fig. 5). The water in the flow loop was seeded with silvercoated hollow glass spheres. The cross-stream velocity data were acquired with frequency shifts of 500 and $700 \mathrm{kHz}$ to shift the measurements of the cross-stream velocity component, with a magnitude below $1 \mathrm{~m} / \mathrm{s}$, above the pedestal frequency and to resolve directional ambiguities.

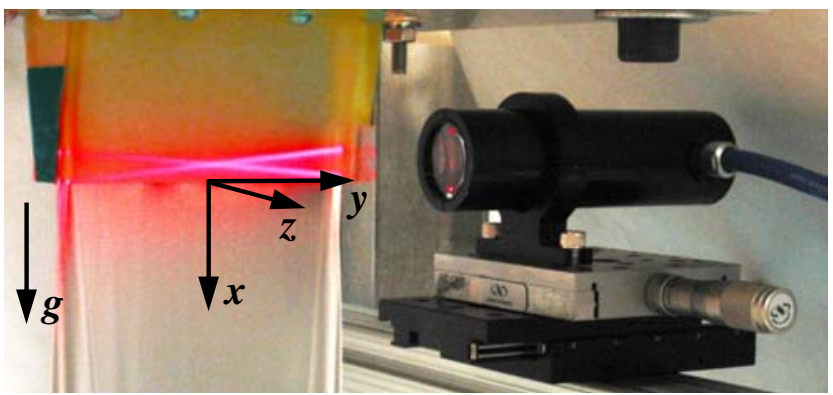

Figure 5 Photo of LDV probe head and nozzle with optical window (shown obtaining $u$ data).

Figure 6 summarizes much of our measurements of $u^{\prime}$ and $w^{\prime}$, the rms fluctuations in the $x$ - and $z$-velocity components, respectively, at $x / \delta=-0.6$ for $R e=120,000$. The graph shows $u_{\text {avg }}^{\prime}$ (open symbols) and $w_{\text {avg }}^{\prime}$ (closed symbols), or these fluctuations spatially averaged over the central $75 \%$ of the $z$-extent of the profile, normalized by the average jet speed, as a function of $y$-position normalized by the $y$-extent of the nozzle at the exit, $W_{\text {o }}$ $\left(W_{0}=10 \mathrm{~cm}\right)$. Results are shown for both the flow conditioner with $(\mathbf{\Delta})$ and without $(\bullet)$ the final screen. Interestingly, the presence or absence of the screen has no discernible impact on the streamwise velocity fluctuations (within experimental error). The absence of the screen does, however, appear to slightly increase the transverse velocity fluctuations, especially near the center of the flow. This result suggests that the transverse velocity fluctuations, or $w^{\prime}$, have a much more significant impact on surface ripple than the streamwise fluctuations. 


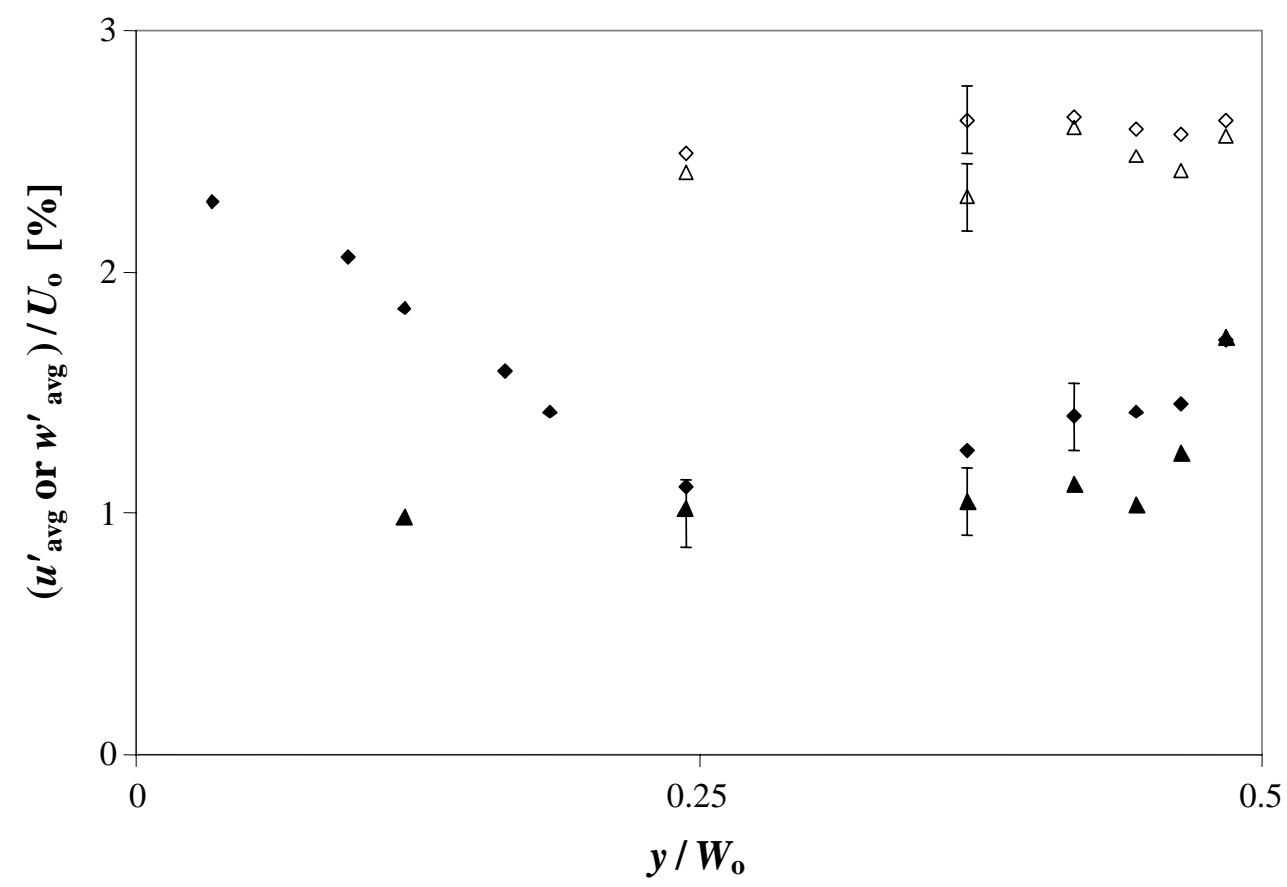

Figure 6 Nondimensional spatially averaged rms velocity fluctuations $u_{\mathrm{avg}}^{\prime} / U_{\mathrm{o}}$ (open) and $w_{\mathrm{avg}}^{\prime} / U_{\mathrm{o}}$ (closed symbols) vs. normalized $y$-position for flow conditioning with $(\boldsymbol{\Delta})$ and without $(\bullet)$ the screen.

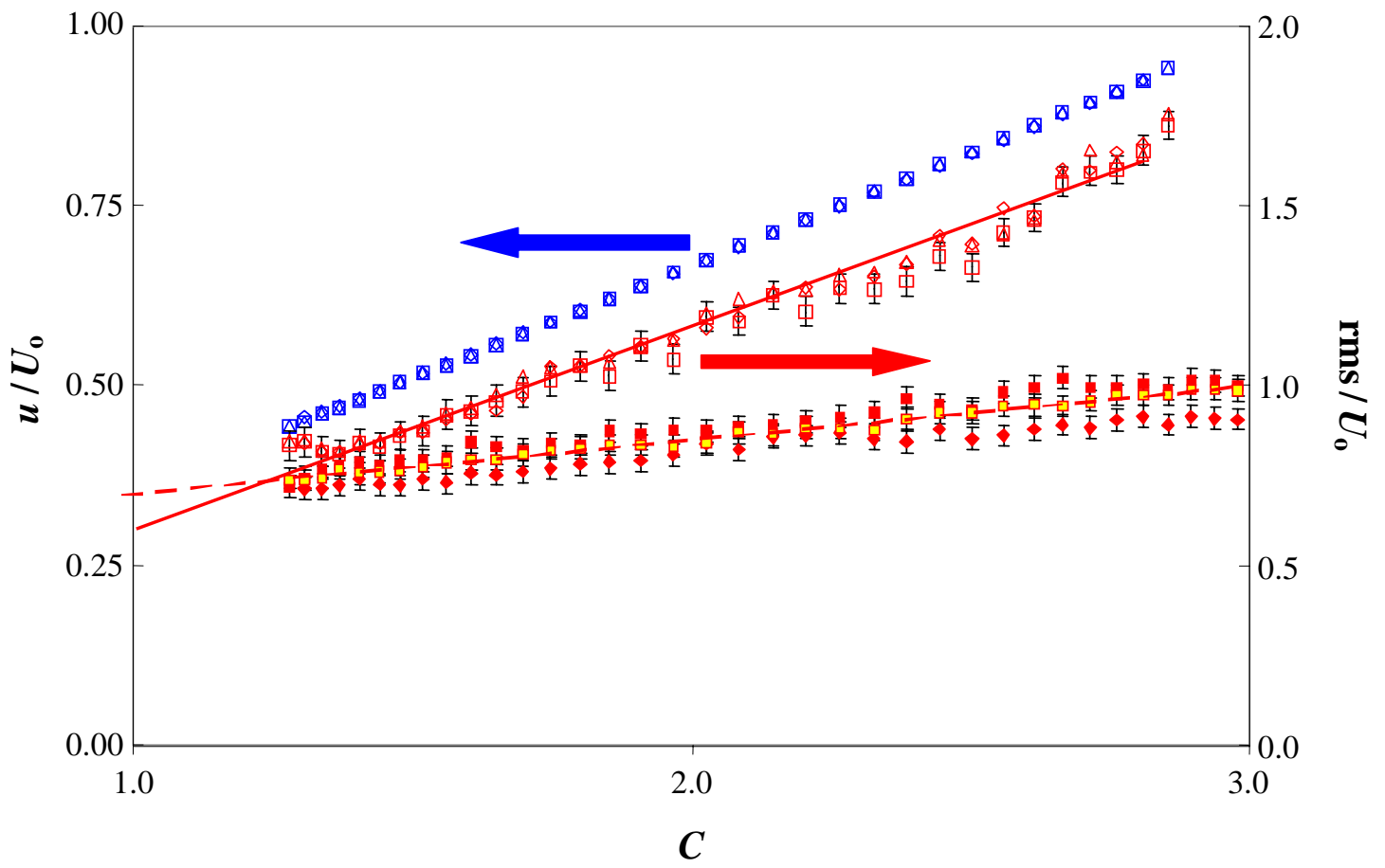

Figure 7 Normalized mean and rms velocity profiles as a function of contraction coefficient $C$ for $R e=$ 120,000 along $z=0$.

Figure 7 shows mean velocity and rms velocity fluctuation profiles normalized by $U_{0}$ as a function of the contraction coefficient $C$ for $R e=120,000$ along $z=0$. The contraction coefficient is the half-width (z-dimension) of the nozzle at its upstream end, or $1.5 \mathrm{~cm}$, 
divided by that at a given $x$-location; $C=1.0$ then corresponds to the upstream end of the nozzle, or $x=-6.3 \mathrm{~cm}$, while $C$ reaches its maximum value of 3.0 at the nozzle exit, or $x$ $=0$. The normalized streamwise mean and rms velocity components are denoted by $u / U_{\text {o }}$ (blue open symbols) and $u^{\prime} / U_{\text {o }}$ (red open symbols), respectively; the normalized rms fluctuations in the cross-stream component are denoted by $w^{\prime} / U_{\mathrm{o}}$ (red filled symbols; different symbol types denote different shift frequencies and $y$-locations). The mean cross-stream velocity, $w=0$ across the nozzle, as expected, so no $w$ data are shown here. As expected, both the mean velocity and its rms fluctuations vary linearly with $C$.

\section{Publications and Presentations}

1) Durbin, S. G., Yoda, M., Abdel-Khalik, S. I., Sadowski, D. L. and Koehler, T. P. "Assessment and control of primary turbulent breakup of thick liquid sheets in IFE reactor cavities - the 'hydrodynamic source term'," Fusion Science and Technology 47, 16-26 (2005)

2) Abdel-Khalik, S. I. and Yoda, M. "An overview of Georgia Tech studies on the fluid dynamics aspects of liquid protection schemes for fusion reactors," Fusion Science and Technology 47, 601-609 (2005) [Invited presentation at the $16^{\text {th }}$ Technology of Fusion Energy Meeting, Madison, WI (2004)]

3) Durbin, S. G., Yoda, M. and Abdel-Khalik, S. I. "Impact of boundary-layer cutting and flow conditioning on free-surface behavior in turbulent liquid sheets," Fusion Science and Technology 47, 718-723 (2005) [Also presented at the $16^{\text {th }}$ Technology of Fusion Energy Meeting, Madison, WI (2004)]

4) Durbin, S. G., Yoda, M. and Abdel-Khalik, S. I. "Flow conditioning design in thick liquid protection,” Fusion Science and Technology 47, 724-728 (2005) [Also presented at the $16^{\text {th }}$ Technology of Fusion Energy Meeting, Madison, WI (2004)]

5) Koehler, T. P., Shin, S., Yoda, M. and Abdel-Khalik, S. I. "Experimental and numerical invesitigation of thin film dry-out analysis for liquid-protected divertors," $21^{\text {st }}$ IEEE/NPSS Symposium on Fusion Engineering (SOFE05), Knoxville, TN (2005)

6) Durbin, S. G. Dynamics and Free-Surface Geometry of Turbulent Liquid Sheets. Ph.D. thesis, Georgia Institute of Technology (2005)

\section{Graduate Students Supported by Grant}

- Samuel G. Durbin (DOE Fusion Energy Sciences Fellowship recipient): Ph.D. received May 2005; currently at Sandia National Laboratories (Albuquerque, NM) in the Experiments and New Programs Group, Organization 6863.

- Timothy P. Koehler: M.S. thesis received Spr 04; Ph.D. qualifying exams completed Spr 05; Ph.D. proposal Fall 05. 


\section{Professional Leadership Activities}

- Dr. Abdel-Khalik was elected to serve as Chair of the Fusion Energy Division of the American Nuclear Society for 2005-06.

- Dr. Yoda was elected to the Executive Committee of the Fusion Energy Division of the American Nuclear Society for 2005-08.

- Dr. Yoda was selected to be a member of the 2006-07 Defense Science Study Group of the Institute for Defense Analyses (Alexandria, VA).

${ }^{1}$ R. W. Moir (2001) “Chamber, target and final focus integrated design,” Nucl. Instrum. Meth. A 464, 140

2 J. F. Latkowski and W. R. Meier (2001) “Heavy-ion fusion final focus magnet shielding designs," Fusion Technol. 39, 798

3 S. G. Durbin (2005) Dynamics and Free-Surface Geometry of Turbulent Liquid Sheets. Ph.D. thesis, Georgia Institute of Technology 